\title{
Spondylitis-psoriasis-enthesitis-enterocolitis-dactylitis-uveitis-peripheral synovitis (SPEED-UP) treatment
}

Brüner $\mathrm{M}^{1,2}$, Dige $\mathrm{A}^{1,3}$, Anne Gitte Loft ${ }^{1,4}$, Trine Bay Laurberg ${ }^{1,4}$, Jørgen Steen Agnholt ${ }^{1,3}$, Kåre

Clemmensen $^{5}$, Iain McInnes ${ }^{6}$, Rik Lories ${ }^{7}$, Lars Iversen ${ }^{1,8}$, Hjuler $\mathrm{KF}^{1,8^{*}}$, Kragstrup TW ${ }^{1,2,4^{*}}$

1) National Center of Autoimmune Diseases, Aarhus University Hospital, 2) Department of Biomedicine, Aarhus University, 3) Department of Hepatology and Gastroenterology, Aarhus University Hospital, 4) Department of Rheumatology, Aarhus University Hospital, 5) Department of Ophthalmology, Aarhus University Hospital 6) Institute of Infection, Immunity and Inflammation, University of Glascow, 7) Skeletal Biology and Engineering Research Center, KU Leuven, 8) Department of Dermatology, Aarhus University Hospital, Aarhus, Denmark

Mads Brüner; 201610201@post.au.dk

Anders Kirch Dige; andedige@rm.dk

Anne Gitte Loft; Anne.Gitte.Loft@auh.rm.dk

Trine Bay Laurberg; trinlaur@rm.dk

Jørgen Steen Agnholt; jorgen.agnholt@aarhus.rm.dk

Kåre Clemmensen; kaarclem@rm.dk

Iain McInnes; Iain.McInnes@glasgow.ac.uk

Rik Lories; rik.lories@kuleuven.be

Lars Iversen; lars.iversen@clin.au.dk

Kasper Fjellhaugen Hjuler; dochjuler@gmail.com

Tue Wenzel Kragstrup; kragstrup@biomed.au.dk

\section{*Corresponding author}

MD PhD Tue Wenzel Kragstrup, Department of Biomedicine, Skou Building, Høegh-Guldbergs Gade 10, Aarhus University, DK-8000 Aarhus C, Denmark, Email: kragstrup@biomed.au.dk, Phone: +4587167265

MD PhD Kasper Fjellhaugen Hjuler; dochjuler@gmail.com

The published version of this article can be found at https://doi.org/10.1016/j.autrev.2020.102731. Full citation: Autoimmune Reviews. 2021 Feb; 20(2):102731 


\begin{abstract}
Axial spondyloarthritis (axSpA), psoriatic arthritis (PsA), psoriasis, inflammatory bowel disease (IBD), and noninfectious uveitis form a distinct group among the immune mediated inflammatory diseases. Thus, many patients suffer from more than one of these disease manifestations. Here, we will use the term spondylitis-psoriasis-enthesitis-enterocolitis-dactylitis-uveitis-peripheral synovitis (SPEED-UP) spectrum disease. The aim is to review the new targeted pharmacological treatment options for all these diseases. All biological or targeted synthetic drugs with U.S. Food and Drug Administration (FDA) or European Medicines Agency (EMA) approval for any of the diagnoses axSpA, PsA, psoriasis, IBD, or non-infectious uveitis were included. Some of the drugs have documented efficacy in more than one of the diseases, e.g. tumor necrosis factor (TNF) inhibitors. However, other drugs are particularly effective for a specific inflamed tissue and approved in only one or two of the disease entities, e.g. abatacept for peripheral arthritis and vedolizumab for inflammatory bowel disease. This contributes with bedside to bench understanding of the immunology underlying this disease spectrum and provides clinicians with an overview that can assist stratified treatment decisions. We hope that this review will help guide clinicians to speed up treatment of patients with this disease spectrum.
\end{abstract}

\title{
Highlights
}

- A stratified approach considering all disease entities in patients affected by more than one disease in the spondylitis-psoriasis-enthesitis-enterocolitis-dactylitis-uveitis-peripheral synovitis spectrum can speed up the time to effective treatment

- Clinical trials with targeted drugs give valuable bedside-to-bench knowledge about similarities and differences in the underlying immunological mechanisms of this disease spectrum

\section{Key words}

Autoimmunity, immune mediated inflammatory diseases, treatment, disease modifying antirheumatic drugs, immunosuppressive drugs, biologic drugs 


\section{Introduction}

Immune-mediated inflammatory diseases (IMIDs) affect up to $10 \%$ of the western population. ${ }^{1}$ Among these diseases, axial spondyloarthritis (axSpA), psoriatic arthritis (PsA), psoriasis, inflammatory bowel disease (IBD), and noninfectious uveitis form a distinct group. ${ }^{2}$ Firstly, many patients exhibit overlapping clinical features commensurate with these diagnoses. ${ }^{3-5}$ Secondly, numerous newer targeted immunosuppressive drugs have demonstrated efficacy in more than one of the diseases, e.g. tumor necrosis factor (TNF) inhibitors. This implies that there are shared immunological pathways involved. However, some of the immunosuppressive drugs are particularly effective for a specific inflamed tissue and approved in only one or two of the diseases, e.g. abatacept for peripheral arthritis, and vedolizumab for inflammatory bowel disease. Therefore, distinct immunological pathways may also be present in each of the diseases, making the management of patients with more than one of these diseases very challenging. Further adding to the complexity are regulatory requirements and pharmaceutical business decisions resulting in medicines not being currently approved for certain diseases despite evidence of efficacy.

Although an association between these diseases is well known, several challenges currently exist in providing high-quality care for these patients. ${ }^{6}$ Firstly, treatment algorithms from national and international societies often focus on one of the diagnoses. Secondly, management of patients is often organized via a monodisciplinary and siloed health care approach. These shortcomings may lead to inefficient management of the individual patient.

The special disease entities of patients with this group of immune mediated inflammatory diseases are psoriasis in the skin, peripheral and axial arthritis, dactylitis, enthesitis, and immune mediated enterocolitis and uveitis. Some immunological alterations manifest as only one disease entity while other immunological alterations can manifest as two or more disease entities. In this paper, we will use the term spondylitis-psoriasis-enthesitis-enterocolitis-dactylitisuveitis-peripheral synovitis (SPEED-UP) spectrum disease. The term is used to facility easy reference to the long list of diseases throughout the manuscript. However, the abbreviation also refers to the need to move this field forward at pace given the range of new treatment options available. The focus of this paper is to review the pharmacological treatment options for all these diseases and provide clinicians with an overview that provides stratified treatment decisions. 


\section{Methods}

The diseases axSpA, psoriasis, PsA, IBD, and non-infectious uveitis were included in this review (Table 1). axSpA was separated into radiographic axSpA (r-axSpA; ankylosing spondylitis (AS)) and non-radiographic axSpA (nr-axSpA). The primary disease entity evaluated in psoriasis was chronic plaque psoriasis (henceforth "psoriasis") with nail psoriasis as a secondary entity. The primary disease entity evaluated in PsA was peripheral synovitis/arthritis with secondary entities being axial arthritis, enthesitis, dactylitis and radiographic progression. IBD was separated into Crohn's disease (CD) and ulcerative colitis (UC) with fistulating CD as a secondary entity. Noninfectious uveitis here covers HLA-B27 associated uveitis and uveitis in association with HLA-B27 negative arthritis, Bechet's disease, sarcoidosis as well as idiopathic intermediate, posterior and panuveitis (Table 1).

Any biological or targeted synthetic drug with a U.S. Food and Drug Administration (FDA) or European Medicines Agency (EMA) approval for any of the diagnoses axSpA, psoriasis, PsA, IBD, or non-infectious uveitis were included. These were the four TNF $\alpha$ specific monoclonal antibodies adalimumab, certolizumab, infliximab, and golimumab, and the TNF-receptor analogue, etanercept; The interleukin 12/23 (IL-12/23) (p40) specific monoclonal antibody ustekinumab , and the three IL-23 (p19) specific monoclonal antibodies guselkumab, risankizumab, and tildrakizumab; The two IL-17A specific monoclonal antibodies secukinumab and ixekizumab; The IL-17 receptor A (IL-17RA) specific monoclonal antibody brodalumab; The cytotoxic-T-lymphocyte-associated protein 4 (CTLA4) analogue (inhibiting cluster of differentiation 80/86 (CD80/86)) abatacept; The $\alpha 4 \beta 7$ integrin specific monoclonal antibody vedolizumab; The phosphodiesterase 4 (PDE4) inhibitor apremilast, and finally the Janus kinase (JAK) inhibitor tofacitinib.

Approval documents from FDA and EMA for each included drug were examined for efficacy demonstrated for the primary indications and any secondary disease entities included in this review. If the documents lacked information concerning the secondary disease entities included in this review, the trials in the FDA and EMA approval documents were studied. If a drug was only approved for some of the disease entities included in this review, a literature review on the use of the drug in the other disease entities was conducted using PubMed. The search term was a combination of disease terms and drug terms. A hand-search was done on reference lists of relevant reviews to identify additional studies. Ongoing and previous/terminated trials were found using clinicaltrials.gov. The search included records from database inception to July $13^{\text {th }} 2020$, which was the date of last search. 
Study selection was by screening records for relevance according to title and abstract. Inclusion criteria were randomized controlled trials (RCTs), prospective and retrospective cohort studies and case series concerning biologic and targeted synthetic disease modifying antirheumatic drugs. Prospective and retrospective cohort studies and case series were excluded if any RCT data existed on the disease and drug combination.

The drugs were then divided in five categories (Table 1). 1) EMA or FDA approved. 2) Primary endpoints met in $\geq$ Phase 2 studies. 3) Contraindicated or to be used with caution. 4) Primary endpoint not met in $\geq$ Phase 2 studies. 5) No data or data from trials below phase 2 .

\section{Results}

\subsection{TNFo inhibitors}

\subsubsection{Adalimumab}

Adalimumab is approved for all the primary disease entities r-axSpA, nr-axSpA, psoriasis, PsA, $\mathrm{CD}, \mathrm{UC}$ and non-infectious uveitis by EMA. ${ }^{7}$ Adalimumab is also approved for all the major entities except for nr-axSpA by FDA. ${ }^{8}$ In psoriasis, adalimumab has demonstrated a treatment benefit in specific psoriasis locations including nail and hand and/or foot psoriasis. $^{7}$

\subsubsection{Certolizumab Pegol}

Certolizumab Pegol is approved by EMA for the treatment of r-axSpA, nr-axSpA, psoriasis and PsA. ${ }^{9}$ Certolizumab Pegol is approved by FDA for r-axSpA, nr-axSpA, psoriasis, PsA, and $\mathrm{CD}^{10}$, but not UC. Concerning non-infectious uveitis, certolizumab has been studied in a phase III trial with r-axSpA patients showing a decreased rate of non-infectious uveitis flares ( 3 per 100 patientyears) compared with placebo (10.3 per 100 patient-years $)^{11}$.

\subsubsection{Infliximab}

Infliximab is approved for $\mathrm{r}-\mathrm{axSpA}$, psoriasis, PsA, CD and $\mathrm{UC}$ by EMA ${ }^{12}$ and FDA ${ }^{13}$. Infliximab is currently the only anti-TNF $\alpha$-antibody approved for the treatment of fistulizing CD. Three entities have positive phase three data without approval: There are positive data concerning early $\mathrm{r}$ axSpA (patients with both r-axSpA and nr-axSpA ${ }^{14}$ ), but there are no studies in dedicated nraxSpA. The drug has been shown to decrease the incidence of non-infectious uveitis in r-axSpA in retrospective trials ${ }^{15}$. Lastly, infliximab has demonstrated a treatment benefit in other psoriasis forms/locations including nail psoriasis. ${ }^{12}$ 


\subsubsection{Golimumab}

Golimumab is approved for the treatment of r-axSpA, nr-axSpA, PsA and UC by EMA ${ }^{16}$ and for the treatment of r-axSpA, PsA and UC by FDA ${ }^{17}$. Golimumab has demonstrated an effect on psoriasis in PsA phase 3 trials $^{18}$ but is currently not approved for psoriasis by EMA or FDA. In an open label phase 4 study examining the extra-articular manifestations of patients with r-axSpA, golimumab reduced the rate of non-infectious uveitis from 11.1 to 2.2 per 100 patient-years ${ }^{19}$.

\subsection{TNF $\alpha / \beta$ inhibitors}

\subsubsection{Etanercept}

Etanercept is approved for the treatment of r-axSpA, nr-axSpA, psoriasis and PsA by EMA ${ }^{20}$, and for the treatment of r-axSpA, psoriasis, and PsA by FDA ${ }^{21}$. No efficacy on non-infectious uveitis has been indicated in RCTs ${ }^{22,23}$. Etanercept has been tested in other psoriasis locations including scalp psoriasis ${ }^{24}$ and open-label trials in nail psoriasis ${ }^{25,26}$ but these secondary disease manifestations have not been specifically approved by the authorities. Etanercept failed to demonstrate efficacy in the treatment of $\mathrm{CD}^{27}$. There are no trials with etanercept in UC.

\subsection{IL-12/23 (p40) inhibitors}

\subsubsection{Ustekinumab}

Ustekinumab is approved for the treatment of psoriasis, PsA, CD and UC by EMA ${ }^{28}$ and FDA ${ }^{29}$. Additionally, ustekinumab has demonstrated a treatment benefit in nail psoriasis ${ }^{28,30}$. In three recent placebo-controlled trials, no efficacy of ustekinumab in the treatment of r-axSpA was demonstrated. ${ }^{31}$ Concerning non-infectious uveitis, one non-industry phase 2 clinical study of ustekinumab for active sight-threatening non-infectious uveitis was recently withdrawn (NCT01647152). There are two other non-industry phase II clinical trials studying the efficacy of ustekinumab in non-infectious uveitis still recruiting (NCT02911116, NCT03847272).

\subsection{IL-23 (p19) inhibitors \\ 3.4.1 Guselkumab}

Guselkumab is approved for the treatment of psoriasis by EMA ${ }^{32}$ and $\mathrm{FDA}^{33}$, and was recently approved for PsA treatment by FDA. ${ }^{33}$ Guselkumab has demonstrated a treatment benefit in scalp, hand and foot psoriasis and nail psoriasis. There are no current trials of guselkumab concerning raxSpA, nr-axSpA, non-infectious uveitis or IBD. 


\subsubsection{Risankizumab}

Risankizumab is approved for the treatment of psoriasis by $\mathrm{EMA}^{34}$ and FDA ${ }^{35}$. Risankizumab failed to meet the primary endpoint of a phase 2 study in biologic naïve patients with $\mathrm{r}-\mathrm{axSpA} \mathrm{S}^{36}$. Concerning PsA, a recently completed phase two trial proved the drug significantly efficacious on peripheral arthritis compared to placebo, but only numerically more efficacious in dactylitis and enthesitis. ${ }^{37}$ Furthermore, one trial in PsA is recently completed with data yet to be reported (NCT02719171) and two trials are ongoing (NCT03675308, NCT03671148). Risankizumab has been shown to induce clinical remission in $31 \%$ of treated CD patients in a recent phase 2 study $^{38}$. There are no current studies of risankizumab in non-infectious uveitis or UC.

\subsubsection{Tildrakizumab}

Tildrakizumab is approved for the treatment of psoriasis by $\mathrm{EMA}^{39}$ and $\mathrm{FDA}^{40}$. The drug is currently being investigated in patients with either r-axSpA or nr-axSpA (NCT02980705, NCT03552276) as well as in PsA patients (NCT03552276, NCT02980692). There are no current studies of tildrakizumab in CD, UC or non-infectious uveitis.

\subsection{IL-17A inhibitors}

\subsubsection{Secukinumab}

Secukinumab is approved for the treatment of $r-a x S p A, n r-a x S p A$, psoriasis and PsA by FDA ${ }^{41}$, while EMA approval extends to nail psoriasis as well ${ }^{42}$. A phase $3 \mathrm{~b}$ trial has reported significant efficacy on axial manifestations of PsA. ${ }^{43}$ In three phase 3 clinical trials of 118 patients with Bechet's uveitis (SHIELD study), 31 patients with active non-infectious uveitis (INSURE study) and 125 patients with quiescent non-infectious uveitis (ENDURE study), subcutaneous secukinumab did not significantly reduce rates of non-infectious uveitis flares. ${ }^{44}$ Another phase II clinical trial in non-infectious uveitis using intravenous secukinumab dosed at either 30 or $10 \mathrm{mg} / \mathrm{kg}$ showed increased response rates compared with the standard $300 \mathrm{mg}$ subcutaneous dose $(72.7,61.5$, and $33.3 \%$, respectively ${ }^{45}$. Secukinumab failed to show any efficacy in the treatment of CD and was even associated with worsening of disease activity in patients with elevated inflammatory parameters $^{46}$.

\subsubsection{Ixekizumab}

Ixekizumab is approved for the treatment of r-axSpA, nr-axSpA, psoriasis and PsA by FDA ${ }^{47}$, while EMA approval extends to nail psoriasis as well ${ }^{48}$. Ixekizumab has additionally demonstrated a 
Running title: SPEED-UP treatment

treatment benefit in specific psoriasis locations including scalp, palmoplantar and genital psoriasis $^{49}$. There are no current trials of ixekizumab in IBD or non-infectious uveitis.

\subsection{IL-17RA inhibitors}

\subsubsection{Brodalumab}

Brodalumab is approved for the treatment of psoriasis by EMA ${ }^{50}$ and FDA $^{51}$. Brodalumab has demonstrated a treatment benefit in other psoriasis locations including nail and scalp psoriasis ${ }^{50}$. Brodalumab treatment has demonstrated efficacy in Asian r-axSpA and nr-axSpA patients in a phase 3 clinical trial ${ }^{52}$. Concerning PsA, the phase 3 trials AMVISION-1 and -2 showed improvements in peripheral arthritis after 24 weeks. ${ }^{53}$ A phase 2 study showed efficacy of brodalumab in patients with PsA at week 12 and indicated sustained response through week 52. One treatment arm significantly improved BASDAI scores, indicating some benefit on PsA-related axial arthritis. ${ }^{54}$ A phase 4 study on several psoriatic entities in the Japanese population (NCT04183881) has been completed but not yet reported. However, the drug is currently not approved for PsA or axSpA by EMA or FDA. Brodalumab treatment in patients with active CD was associated with worsening of disease activity and treatment should be avoided in patients with known $\mathrm{CD}^{55}$. There are no current trials of brodalumab in UC or non-infectious uveitis.

\subsection{CD80/86 inihibitors}

\subsubsection{Abatacept}

Abatacept is approved for PsA by both $\mathrm{EMA}^{56}$ and FDA ${ }^{57}$. Only one of the pivotal trials investigated dactylitis and enthesitis. This study failed to reach a significantly higher fraction of patients with complete resolution even though the proportions were numerically higher in the active treatment arms than in the placebo $\operatorname{arms}^{58}$. There was no response reported in a prospective openlabel pilot r-axSpA study ${ }^{59}$. No significant effect of abatacept was seen on psoriasis in the PsA phase 3 trials $^{56,58}$. Abatacept has failed to show efficacy in the treatment of CD and $\mathrm{UC}^{60}$. There are no current trials of abatacept in non-infectious uveitis.

\section{8 $\alpha 4 \beta$ 7-integrin inhibitors}

\subsubsection{Vedolizumab}

Vedolizumab is approved for the treatment of CD and UC by both $\mathrm{EMA}^{61}$ and FDA ${ }^{62}$. Significant efficacy on fistulizing CD has been reported ${ }^{63}$. There has been several case reports of flare or new occurrence of rheumatic disorders in patients treated with vedolizumab ${ }^{64-66}$. However, in a recent 
cohort of IBD patients treated with vedolizumab no case of induction or flare of arthritis and/or sacroiliitis was reported. Indeed, 6 out of the 14 patients with active $\operatorname{SpA}(46.2 \%)$-all complaining of peripheral arthropathy - experienced clinical benefit after the initiation of vedolizumab ${ }^{67}$. There are no trials of vedolizumab in SpA, psoriasis, PsA, or non-infectious uveitis.

\subsection{PDE4 inhibitors}

\subsubsection{Apremilast}

Apremilast is approved for the treatment of PsA and psoriasis by EMA ${ }^{68}$ and FDA ${ }^{69}$. In addition, apremilast has demonstrated a treatment benefit in scalp and nail psoriasis. ${ }^{68}$ In a phase II study over 12 weeks in 38 patients with symptomatic r-axSpA, apremilast did not meet the primary end point $^{70}$. A larger phase 3 trial including 490 patients have still not been published but preliminary reports suggest a failure of apremilast to meet the primary end point (NCT01583374). A randomized clinical trial evaluating the efficacy of apremilast for the treatment of non-infectious uveitis has been terminated due to lack of efficacy in three subjects enrolled (NCT00889421). There are no trials of apremilast in UC or CD.

\subsection{JAK inhibitors}

\subsubsection{Tofacitinib}

Tofacitinib is approved for the treatment of PsA and UC by both EMA ${ }^{71}$ and FDA ${ }^{72}$. A phase II dose-ranging trial has shown efficacy of tofacitinib in reducing the signs, symptoms and spinal inflammation of r-axSpA in adults with active disease ${ }^{73}$. Tofacitinib has demonstrated significant efficacy vs. placebo in patients with psoriasis in two pivotal phase III trials ${ }^{74}$. A further psoriasis? phase 3 trial showed non-inferiority of tofacitinib $10 \mathrm{mg}$ twice daily to etanercept $50 \mathrm{mg}$ twice weekly, whereas tofacinitib $5 \mathrm{mg}$ twice daily did not show non-inferiority to etanercept $50 \mathrm{mg}$ twice weekly ${ }^{75}$. Tofacitinib is currently not approved for psoriasis by EMA or FDA. A phase II clinical trial on non-infectious uveitis is currently registered but not yet recruiting (NCT03580343).

Tofacitinib has failed to show efficacy in the treatment of $\mathrm{CD}^{76}$.

\section{Discussion}

The disease entities spondylitis, psoriasis, arthritis, dactylitis, enthesitis, enterocolitis, and noninfectious uveitis are a group of associated IMIDs with shared immunopathology, in which specific targeted immunosuppressive drugs have demonstrated efficacy in more than one of the diseases. 
Here, we use the term SPEED-UP (spondylitis-psoriasis-enthesitis-enterocolitis-dactylitis-uveitisperipheral synovitis) spectrum disease as stated in the introduction. In patients with more than one disease entity the clinical management should therefore, depending on the clinical expression, use an interdisciplinary approach that focus the treatment choice on efficacy in the SPEED-UPconditions of relevance to the specific patients. These patients experience time consuming multiple clinic visits in different specialties, which neglect some disease manifestations/entities? and the patients are left on their own with the responsibility for coordinating the different treatment approaches $^{6,77}$. This review emphasizes the need of an interdisciplinary approach concerning patients with SPEED-UP spectrum disease.

As with any IMID, this SPEED-UP spectrum disease is caused by not yet clearly defined alterations in the immune system. Some alterations are associated with one disease entity only while others may cause two or more disease manifestations. Viewing the diseases as caused by common immunological pathways with a variation in clinical phenotype could be an important change in the perception and evaluation of the diseases and lead to more patient centered management. Here, we review the pharmacological treatment options for all entities in the SPEEDUP spectrum disease. Any biological or targeted synthetic drug with FDA or EMA approval for any of the diagnoses axSpA, psoriasis, PsA, IBD, or non-infectious uveitis was included. Hopefully, this will facilitate the clinicians' opportunities to deliver a stratified and tailored treatment (Table 1, Figure 1).

All five TNF inhibitors are approved for one or more entities of SPEED-UP spectrum disease (Figure 1). TNF $\alpha$ is primarily released by macrophages ${ }^{78}$ and plays a crucial role in proinflammatory activity. ${ }^{79}$ Adalimumab, infliximab, golimumab, and certolizumab all target both soluble and membrane bound TNF $\alpha$. Adalimumab, golimumab, and infliximab are all monoclonal antibodies. Certolizumab pegol is a pegylated anti-TNF $\alpha$ fab fragment with prolonged half-life. ${ }^{80}$ Etanercept is a soluble TNF $\alpha$-receptor analogue and targets both soluble TNF $\alpha$ and lymphotoxin but shows decreased binding capacity to the membrane bound forms. All five TNF $\alpha$ inhibitors are approved for many of the SPEED-UP disease entities. In this way TNF $\alpha$ seems to be a major driver of SPEED-UP spectrum disease. However, studies of the TNF $\alpha$-receptor analogue etanercept in IBD and non-infectious uveitis did not meet the primary end points ${ }^{22,27}$. Additionally, numerically lower response rates are reported for etanercept in psoriasis compared to the other TNFa inhibitors $^{81}$. This could possibly be due to the less pronounced binding of etanercept to membrane bound TNF $\alpha$. Monoclonal TNF $\alpha$ inhibitor antibodies binding to membrane bound TNF $\alpha$ has been 
reported to induce immune cell apoptosis in IBD in an autocrine fashion, a treatment effect considered of pivotal importance in IBD. ${ }^{82-85}$ Therefore, etanercept is not used in patients with significant IBD symptoms as a part of the SPEED-UP disease spectrum. Importantly, the monoclonal TNF $\alpha$ inhibitor antibodies are the only drugs approved for the treatment of noninfectious uveitis. Importantly, TNF $\alpha$ inhibitors are not a one size fits all despite the broad range of effects and the largest number of approvals. As mentioned in the following sections, other drugs are superior to TNF $\alpha$ inhibitors in certain disease manifestations.

Concerning inhibition of the IL-12/23 axis, a single IL-12/23 inhibitor and three IL-23 inhibitors are approved for one or more entities of SPEED-UP spectrum disease (Figure 1). IL-23 is a master regulator of Th17 activity ${ }^{86}$ and maintains Th17 cell expansion and a prolonged upregulation of IL-17A secretion ${ }^{87,88}$. Ustekinumab is an IL-12/23 (p40) specific monoclonal antibody and is approved for psoriasis, PsA and IBD. Ustekinumab has shown superior efficacy compared with the $\mathrm{TNF} \alpha / \beta$ inhibitor etanercept in head-to-head trials in psoriasis ${ }^{89}$. In contrast, recent trials of ustekinumab in axSpA did not meet the primary endpoints. Also, efficacy on peripheral arthritis appears to be numerically smaller for ustekinumab compared with TNF inhibitors. IL-12/23 inhibition (ustekinumab) has also been approved for the treatment of both CD and UC. Ustekinumab has not been considered the first-line treatment choice in bio-naïve IBD patients since there has been no head to head trials with infliximab. However, in patients not responding to TNF inhibitors, ustekinumab has proved effective. Therefore, ustekinumab treatment may be considered for patients with IBD as the only manifestation and in case of co-occurence of psoriasis/PsA. The three IL-23 (p19) specific monoclonal antibodies guselkumab, risankizumab, and tildrakizumab are all approved for psoriasis and guselkumab was recently approved for PsA. These drugs have shown superior efficacy compared with some of the TNF inhibitors in head-tohead trials in psoriasis, as guselkumab and risankizumab have achieved significantly higher PASI90 scores than adalimumab ${ }^{90,91}$, and tildrakizumab has achieved a significantly higher PASI90 score than etanercept. ${ }^{92}$ Meanwhile, IL-23 (p19) specific inhibition failed in axSpA trials. ${ }^{36}$ Therefore, IL23 inhibition might not be first choice in patients with axSpA. The trials of the IL-23 (p19) specific monoclonal antibodies in PsA are ongoing and will show whether the effect on peripheral arthritis is numerically closer to ustekinumab or TNF inhibition. The lack of effect of IL-23 inhibition in axSpA is surprising considering the role of IL-23 in the IL-23-Th17 axis. However, IL-23 has other functions and IL-17A is secreted by many other cell types than Th17 cells. Collectively, IL-12/23 
and IL-23 inhibition is efficacious in many SPEED-UP spectrum entities but will likely not be first choice in patients with axSpA involvement ${ }^{93}$.

Three inhibitors of IL-17 cytokines and receptors are approved for one or more entities of SPEED-UP spectrum disease (Figure 1). The IL-23-Th17 axis is a majorly influencing factor in the pathogenesis of SPEED-UP spectrum disease ${ }^{94}$. IL-17A release results in inflammation, matrix destruction, cartilaginous damage and bone erosion. ${ }^{95}$ Secukinumab and ixekizumab are both monoclonal antibodies targeting IL-17A. ${ }^{95,96}$ These drugs are approved for axSpA, psoriasis and PsA. Brodalumab is a monoclonal antibody directed against the IL-17RA. ${ }^{97}$ This drug has so far only been approved for psoriasis. IL-17 inhibition has also shown superiority compared with TNF $\alpha$ inhibition in head-to-head trials in psoriasis. ${ }^{98-100}$ Blocking IL-17A signaling both at the cytokine level and at the receptor level has been shown to be superior to ustekinumab in clinical trials in psoriasis. ${ }^{98,101}$ Head-to-head psoriasis trials have shown superiority of two IL23(p19) inhibitors compared with secukinumab ${ }^{102,103}$, whereas ixekizumab, has shown superiority compared with guselkumab in short-term psoriasis endpoints ${ }^{104}$. This could be because of differences between the IL-17A inhibitors but could also be the result of differences in clinical trial design. Importantly, both ixekizumab and secukinumab were not inferior compared with adalimumab in head-to-head trials in PsA ${ }^{105,106}$. Taken together, IL-17A seems to be more important compared with TNF $\alpha$ in skin disease and more important compared with IL-23 in axial disease in SPEED-UP spectrum disease. In contrast, a trial of IL-17A inhibition with secukinumab in $\mathrm{CD}$ patients showed no effect of the drug and higher rates of disease-worsening, leading to early termination of the trial. ${ }^{46}$ Indeed, IBD related adverse events have been described in trials of IL-17 inhibition in SpA, psoriasis and PsA ${ }^{107}$. Therefore, IL-17 inhibition is not recommended in patients with active IBD as one of the disease manifestations of SPEED-UP spectrum disease.

The CTLA4 analogue (inhibiting CD80/86) abatacept is approved for PsA. T-cell activation requires binding of the co-stimulatory receptor CD28 to CD80/86 on an antigen presenting cell. Abatacept is therefore considered to function via suppression of T cell responses. ${ }^{108}$ Interestingly, inhibition of CTLA4 is being used in oncology to activate CD8 positive T cells against tumor cells. This treatment may in some cases elicit immune mediated adverse events resembling psoriasis skin disease, seronegative arthritis and ulcerative colitis ${ }^{109}$. However, trials with abatacept failed to meet the primary end point in both SpA, psoriasis, and IBD. So, abatacept should only be used in patients with peripheral arthritis. 
The $\alpha 4 \beta 7$ integrin specific monoclonal antibody vedolizumab is approved for the treatment of IBD. The integrin $\alpha 4 \beta 7$ is expressed by a subset of lymphocytes and binds MAdCAM1 on vascular endothelial cells in the gastrointestinal tract. Vedolizumab is believed to be rather specific in preventing migration of gut homing lymphocytes to the lamina propria. The drug has therefore not been studied in other entities of SPEED-UP spectrum disease. Therefore, vedolizumab should only be used in patients with IBD as the dominating disease entity.

The PDE4 inhibitor apremilast is approved for the treatment of psoriasis and PsA. Apremilast is an orally administered, small-molecule drug inhibiting PDE4. PDE4 inhibition mediates a continuously high intracellular cAMP-concentration, thereby inhibiting the production of proinflammatory cytokines (Figure 1). ${ }^{110}$ The main cytokine inhibited by apremilast treatment of synovial fluid mononuclear cells was the IL-12/23 p40 subunit. ${ }^{111}$ Apremilast has shown efficacy primarily in psoriasis. However, PASI75 improvement appears to be numerically smaller with apremilast compared with monoclonal TNF $\alpha$ inhibitor antibodies and biological drugs targeting IL12/23, IL-23, and IL-17. Apremilast is also approved for peripheral PsA. However, the effect is limited on ACR70 responses and radiographic data is still lacking. Thus, apremilast can be used in patients with psoriasis and perhaps mild PsA ${ }^{93}$.

The JAK inhibitor tofacitinib is approved in PsA and IBD (UC only). However, many studies with different JAK inhibitors are ongoing. The intracellular JAK pathway mediates expression of key pro-inflammatory cytokines and growth factors (Figure 1). ${ }^{12}$ Tofacitinib is a JAK $1 / 3$ inhibitor with a limited effect on JAK 2. Some of the cytokines inhibited by tofacitinib are also the targets of other drugs approved for the treatment of SPEED-UP spectrum disease including IL-12 and IL-23. Several other cytokines could be implicated such as IL-2, IL-7, and the IL-20 cytokine family ${ }^{113}$. In UC, tofacitinib often improves symptoms after a few days of treatment. The tofacitinib $10 \mathrm{mg}$ twice daily dose regimen is usually used for three months and subsequently reduced to $5 \mathrm{mg}$ twice daily. Importantly, JAK inhibition is still not approved for psoriasis. This is mainly because the tofacitinib $10 \mathrm{mg}$ twice daily dose has been linked to increased risk of side effects and because the tofacitinib $5 \mathrm{mg}$ twice daily dose was inferior compared with etanercept. However, the tyrosine kinase 2 (TYK2) pathway, another member of the JAK family, seems to be important in psoriasis pathophysiology and selective TYK2 inhibition has shown promising results in a phase 2 trial of psoriasis ${ }^{114}$. The cytokines mediated by the TYK2 signaling pathway include IL-12, IL-23 and the IL-20 cytokine family and could therefore be more specific 
for psoriasis with a better safety profile. Taken together, tofacitinib is still primarily recommended in patients dominated by peripheral arthritis or ulcerative colitis.

There are several limitations in this review. First, the adverse event profiles and the long-term follow-up data on the different drugs were not included. This information will be important for the choice of treatment in many patients. Second, the route of administration and drug prices were not considered. These might be very important factors in many health care systems. Third, non-steroidal anti-inflammatory drugs and conventional synthetic immunosuppressive drugs such as methotrexate, azathioprine, dimethyl fumarate, and leflunomide were not taken into consideration even though combinations with these drugs are indicated in many patients with SPEED-UP spectrum disease. Further, effect sizes of the drugs have not been systematically reported. Therefore, this review cannot be used to position the different products. Finally, we did not discuss switching between drugs. Prior failed medications need to be considered in the treatment decision.

Taken together, we have reviewed the pharmacological treatment options for all entities and manifestations of what we here term in SPEED-UP spectrum disease. Any biological or targeted synthetic drug with FDA or EMA approval for any of the diagnoses axSpA, psoriasis, PsA, IBD, or non-infectious uveitis was included. We hope that this review will help guide clinicians to speed up treatment of patients with these diseases.

\section{Authors' contributions}

$\mathrm{MB}, \mathrm{AD}, \mathrm{KFJ}$, and TWK performed the literature search and wrote the result sections. MBK and TWK made the first draft of the manuscript. All authors were involved in analysis and interpretation of data and critically revising the manuscript for intellectual content. All authors read and approved the final version.

\section{$6 \quad$ Ethics approval and consent to participate}

Not applicable.

\section{Disclosures/conflict of interest:}

TWK has engaged in educational activities receiving speaking fees from Pfizer, Bristol-Myers Squibb, Eli Lilly, Novartis, and UCB and has been consultant and advisor for Bristol-Myers Squibb 
Running title: SPEED-UP treatment

and Gilead. TWK is co-founder and clinical developer in iBiotech ApS developing diagnostic and therapeutic solutions for people with autoimmune diseases and cancer.

JA advisory board for Ferring Pharmaceuticals, AbbVie and Pfizer and speaker and educational sessions for Bristol-Meyers Squibb, Takeda, Norgine, Cilag-Janssen. Consultant for Janssen.

AGL advisory board for AbbVie, Pfizer, MSD, Novartis, Lilly and speaker and educational sessions for MSD, Novartis, Pfizer and UCB.

LI has served as a consultant and/or paid speaker for and/or participated in clinical trials sponsored by: AbbVie, Almirall, Amgen, Astra Zeneca, BMS, Boehringer Ingelheim, Celgene, Centocor, Eli

Lilly, Janssen Cilag, Kyowa, Leo Pharma, MSD, Novartis, Pfizer, Samsung, UCB

KFH has been a consultant and advisor for the following companies: AbbVie, LEO Pharma, Novartis and has received speaking fees from: AbbVie, LEO Pharma, Novartis, Janssen, CSL

Behring.

$\mathrm{KC}$ has served as a paid speaker for Abbvie.

Leuven Research and Development, the technology transfer office of KU Leuven has received consultancy and speaker's fees, and research grants on behalf of RL from Abbvie, Boehringer-Ingelheim, Celgene, Eli-Lilly, Galapagos, Janssen, MSD, Novartis, Pfizer, Samumed and UCB.

\section{Funding}

TWK was supported by a grant from Independent Research Fund Denmark (9039-00015B). The basic costs of establising and running the interdisciplinary clinical centre (Nationalt Center for Autoimmune Sygdomme) are supported by a grant from The Danish Ministry of Health.

\section{Availability of data and material}

Not applicable.

\section{List of abbreviations}

AS: Ankylosing spondylitis

axSpA: Axial spondyloarthritis

CD: Crohns disease

EMA: European Medicines Agency 
FDA: U.S. Food and Drug Administration

IBD: Inflammatory bowel disease

IMID: Immune mediated inflammatory disease

MAb: Monoclonal antibody

Nr-axSpA: Non-radiographic axial spondyloarthritis

PsA: Psoriatic arthritis

r-axSpA: Radiographic axial spondyloarthritis

RCT: Randomized controlled trial

SpA: Spondyloarthritis

UC: Ulcerative colitis

\subsection{References}

1 Bayry, J. \& Radstake, T. R. Immune-mediated inflammatory diseases: progress in molecular pathogenesis and therapeutic strategies. 9, 297-299, doi:10.1586/eci.13.10 (2013).

2 Cohen, R. et al. Autoimmune disease concomitance among inflammatory bowel disease patients in the United States, 2001-2002. Inflamm Bowel Dis 14, 738-743, doi:10.1002/ibd.20406 (2008).

$3 \mathrm{Fu}$, Y., Lee, C.-H. \& Chi, C.-C. Association of Psoriasis With Inflammatory Bowel Disease. JAMA Dermatology 154, 1417, doi:10.1001/jamadermatol.2018.3631 (2018).

4 Scriffignano, S., Perrotta, F. M., De Socio, A. \& Lubrano, E. Role of comorbidities in spondyloarthritis including psoriatic arthritis. Clinical Rheumatology 38, 3-10, doi:10.1007/s10067-018-4332-7 (2019).

5 Rosenbaum, J. T. Uveitis in spondyloarthritis including psoriatic arthritis, ankylosing spondylitis, and inflammatory bowel disease. Clinical Rheumatology 34, 999-1002, doi:10.1007/s10067-015-2960-8 (2015).

6 Haberman, R. et al. Bridging the Gaps in the Care of Psoriasis and Psoriatic Arthritis: the Role of Combined Clinics. Curr Rheumatol Rep 20, 76, doi:10.1007/s11926-018-0785-6 (2018).

7 European Medicines Agency. Humira (Adalimumab) Annex I summary of product characteristics, $<$ https://www.ema.europa.eu/en/documents/product-information/humira-epar-productinformation en.pdf> (2020).

8 U.S. Food \& Drug Administration. Humira (Adalimumab) Supplementary Label 1250570rig1s411/bl, <https://www.accessdata.fda.gov/drugsatfda docs/label/2019/1250570rig1s411lbl.pdf> (2019).

9 European Medicines Agency. Cimzia (Certolizumab Pegol) Annex I summary of product characteristics, <https://www.ema.europa.eu/en/documents/product-information/cimzia-eparproduct-information en.pdf $>$ (2019).

10 U.S. Food \& Drug Administration. Cimzia (Certolizumab Pegol) Supplementary label SUPPL-293, $<$ https://www.accessdata.fda.gov/drugsatfda docs/label/2019/125160s293lbl.pdf> (2019).

11 Rudwaleit, M. et al. Observed Incidence of Uveitis Following Certolizumab Pegol Treatment in Patients With Axial Spondyloarthritis. Arthritis Care Res (Hoboken) 68, 838-844, doi:10.1002/acr.22848 (2016).

12 European Medicines Agency. Remicade (Infliximab) Annex I summary of product characteristics, $<$ https://www.ema.europa.eu/en/documents/product-information/remicade-epar-productinformation en.pdf> (2019).

13 U.S. Food \& Drug Administration. Remicade (Infliximab) Supplementary Label SUPPL-5385, <https://www.accessdata.fda.gov/drugsatfda docs/label/2018/103772s5385lbl.pdf> (2018). 
Sieper, J. et al. Efficacy and safety of infliximab plus naproxen versus naproxen alone in patients with early, active axial spondyloarthritis: results from the double-blind, placebo-controlled INFAST study, Part 1. Annals of the Rheumatic Diseases 73, 101-107, doi:10.1136/annrheumdis-2012203201 (2014).

15 Bodaghi, B. et al. Therapeutic use of infliximab in sight threatening uveitis: retrospective analysis of efficacy, safety, and limiting factors. Ann Rheum Dis 64, 962-964, doi:10.1136/ard.2004.025882 (2005).

16 European Medicines Agency. Simponi (Golimumab) Annex I summary of product characteristics, <https://www.ema.europa.eu/en/documents/product-information/simponi-epar-productinformation en.pdf> (2019).

17 U.S. Food \& Drug Administration. Simponi (Golimumab) Supplementary label SUPPL-146, <https://www.accessdata.fda.gov/drugsatfda docs/label/2019/125289s146lbl.pdf> (2019).

18 Kavanaugh, A. et al. Golimumab, a new human tumor necrosis factor $\alpha$ antibody, administered every four weeks as a subcutaneous injection in psoriatic arthritis: Twenty-four-week efficacy and safety results of a randomized, placebo-controlled study. 60, 976-986, doi:10.1002/art.24403 (2009).

19 Van Bentum, R. E. et al. Reduced Occurrence Rate of Acute Anterior Uveitis in Ankylosing Spondylitis Treated with Golimumab - The GO-EASY Study. The Journal of Rheumatology 46, 153159, doi:10.3899/jrheum.180312 (2019).

20 European Medicines Agency. Enbrel (Etanercept) Annex I summary of product characteristics, $<$ https://www.ema.europa.eu/en/documents/product-information/enbrel-epar-productinformation en.pdf> (2019).

21 U.S. Food \& Drug Administration. Enbrel (Etanercept) Supplementary label SUPPL-5569, $<$ https://www.accessdata.fda.gov/drugsatfda docs/label/2018/103795s5569lbl.pdf> (2018).

22 Foster, C. S. et al. Efficacy of etanercept in preventing relapse of uveitis controlled by methotrexate. Arch Ophthalmol 121, 437-440, doi:10.1001/archopht.121.4.437 (2003). Guignard, S. et al. Efficacy of tumour necrosis factor blockers in reducing uveitis flares in patients with spondylarthropathy: a retrospective study. Annals of the Rheumatic Diseases 65, 1631-1634, doi:10.1136/ard.2006.052092 (2006).

24 Bagel, J. et al. Moderate to severe plaque psoriasis with scalp involvement: a randomized, doubleblind, placebo-controlled study of etanercept. J Am Acad Dermatol 67, 86-92, doi:10.1016/j.jaad.2011.07.034 (2012).

Ortonne, J. P. et al. A 24-week randomized clinical trial investigating the efficacy and safety of two doses of etanercept in nail psoriasis. British Journal of Dermatology 168, 1080-1087, doi:10.1111/bjd.12060 (2013).

Pasch, M. C. Nail Psoriasis: A Review of Treatment Options. Drugs 76, 675-705, doi:10.1007/s40265-016-0564-5 (2016).

27 Sandborn, W. J. et al. Etanercept for active Crohn's disease: a randomized, double-blind, placebocontrolled trial. Gastroenterology 121, 1088-1094, doi:10.1053/gast.2001.28674 (2001). European Medicines Agency. Stelara (Ustekinumab) Annex I summary of product characteristics, $<$ https://www.ema.europa.eu/en/documents/product-information/stelara-epar-productinformation en.pdf> (2019).

U.S. Food \& Drug Administration. Stelara (Ustekinumab) Supplementary label SUPPL-142, $<$ https://www.accessdata.fda.gov/drugsatfda docs/label/2019/125261s142,761044s001lbl.pdf> (2019).

30 Blauvelt, A. et al. Efficacy and safety of guselkumab, an anti-interleukin-23 monoclonal antibody, compared with adalimumab for the continuous treatment of patients with moderate to severe psoriasis: Results from the phase III, double-blinded, placebo- and active comparator-controlled VOYAGE 1 trial. J Am Acad Dermatol 76, 405-417, doi:10.1016/j.jaad.2016.11.041 (2017). 
31 Deodhar, A. et al. Three Multicenter, Randomized, Double - Blind, Placebo - Controlled Studies Evaluating the Efficacy and Safety of Ustekinumab in Axial Spondyloarthritis. Arthritis \& Rheumatology 71, 258-270, doi:10.1002/art.40728 (2019).

32 European Medicines Agency. Tremfya (Guselkumab) Annex I Summary of product characteristics, $<$ https://www.ema.europa.eu/en/documents/product-information/tremfya-epar-productinformation en.pdf> (2020).

33 U.S. Food \& Drug Administration. Tremfya (Guselkumab) Supplementary label <https://www.accessdata.fda.gov/drugsatfda docs/label/2020/761061s007lbl.pdf> (2019). European Medicines Agency. Skyrizi (Risankizumab) Annex I Summary of product characteristics, $<$ https://www.ema.europa.eu/en/documents/product-information/skyrizi-epar-productinformation en.pdf> (2019).

U.S. Food \& Drug Administration. Skyrizi (Risankizumab) Supplementary label ORIG-1, $<$ https://www.accessdata.fda.gov/drugsatfda docs/label/2019/761105s000lbl.pdf> (2019). Baeten, D. et al. Risankizumab, an IL-23 inhibitor, for ankylosing spondylitis: results of a randomised, double-blind, placebo-controlled, proof-of-concept, dose-finding phase 2 study. Ann Rheum Dis 77, 1295-1302, doi:10.1136/annrheumdis-2018-213328 (2018).

37 Mease, P. J. et al. (BMJ Publishing Group Ltd and European League Against Rheumatism).

38 Feagan, B. G. et al. Induction therapy with the selective interleukin-23 inhibitor risankizumab in patients with moderate-to-severe Crohn's disease: a randomised, double-blind, placebo-controlled phase 2 study. Lancet 389, 1699-1709, doi:10.1016/s0140-6736(17)30570-6 (2017).

39 European Medicines Agency. Ilumetri (Tildrakizumab) Annex I Summary of product characteristics, $<$ https://www.ema.europa.eu/en/documents/product-information/ilumetri-epar-productinformation en.pdf> (2019).

40 U.S. Food \& Drug Administration. Ilumya (Tildrakizumab) Supplementary label ORIG-1, $<$ https://www.accessdata.fda.gov/drugsatfda docs/label/2018/761067s000lbl.pdf> (2018). U.S. Food \& Drug Administration. Cosentyx (Secukinumab) Supplementary Label SUPPL-31, $<$ https://www.accessdata.fda.gov/drugsatfda docs/label/2020/125504s031lbl.pdf> (2020). European Medicines Agency. Cosentyx (Secukinumab) Annex I Summary of product characteristics, $<$ https://www.ema.europa.eu/en/documents/product-information/cosentyx-epar-productinformation en.pdf> (2019).

43 Baraliakos, X. et al. (BMJ Publishing Group Ltd and European League Against Rheumatism).

44 Dick, A. D. et al. Secukinumab in the treatment of noninfectious uveitis: results of three randomized, controlled clinical trials. Ophthalmology 120, 777-787, doi:10.1016/j.ophtha.2012.09.040 (2013).

Letko, E. et al. Efficacy and safety of intravenous secukinumab in noninfectious uveitis requiring steroid-sparing immunosuppressive therapy. Ophthalmology 122, 939-948, doi:10.1016/j.ophtha.2014.12.033 (2015).

46 Hueber, W. et al. Secukinumab, a human anti-IL-17A monoclonal antibody, for moderate to severe Crohn's disease: unexpected results of a randomised, double-blind placebo-controlled trial. Gut 61, 1693-1700, doi:10.1136/gutjnl-2011-301668 (2012).

47 European Medicines Agency. Taltz (Ixekizumab) Annex I Summary of product characteristics, $<$ https://www.ema.europa.eu/en/documents/product-information/taltz-epar-productinformation en.pdf> (2019).

48 U.S. Food \& Drug Administration. Taltz (Ixekizumab) Supplementary label, $<$ https://www.accessdata.fda.gov/drugsatfda docs/label/2019/125521s016lbl.pdf> (2019).

49 Ryan, C. et al. Efficacy and safety of ixekizumab in a randomized, double-blinded, placebocontrolled phase IIIb study of patients with moderate-to-severe genital psoriasis. Br J Dermatol 179, 844-852, doi:10.1111/bjd.16736 (2018). 
50 European Medicines Agency. Kyntheum (Brodalumab) Annex I Summary of product characteristics, $<$ https://www.ema.europa.eu/en/documents/product-information/kyntheum-epar-productinformation en.pdf $>$ (2019).

51 U.S. Food \& Drug Administration. Siliq (Brodalumab) Supplementary Label ORIG-1, $<$ https://www.accessdata.fda.gov/drugsatfda docs/label/2017/761032lbl.pdf> (2017).

52 Wei, J. C.-C. et al. (BMJ Publishing Group Ltd and European League Against Rheumatism).

53 Mcinnes, I. M., M.,; Hjuler, K.; Raymond, K.; Helliwell, P.;. Brodalumab in psoriatic arthritis (PsA): 24-week results from the phase III AMVISON-1 and -2 trials. Journal of the American Academy of Dermatology 81, AB28, doi:10.1016/j.jaad.2019.06.140 (2019).

54 Mease, P. J. et al. Brodalumab, an Anti-IL17RA Monoclonal Antibody, in Psoriatic Arthritis. New England Journal of Medicine 370, 2295-2306, doi:10.1056/nejmoa1315231 (2014).

55 Targan, S. R. et al. A Randomized, Double-Blind, Placebo-Controlled Phase 2 Study of Brodalumab in Patients With Moderate-to-Severe Crohn's Disease. Am J Gastroenterol 111, 1599-1607, doi:10.1038/ajg.2016.298 (2016).

56 European Medicines Agency. Orencia (Abatacept) Annex I Summary of product characteristics, $<$ https://www.ema.europa.eu/en/documents/product-information/orencia-epar-productinformation en.pdf> (2020).

57 U.S. Food \& Drug Administration. Orencia (Abatacept) Supplementary Label SUPPL-224, $<$ https://www.accessdata.fda.gov/drugsatfda docs/label/2019/125118s224lbl.pdf> (2019).

58 Mease, P. J. et al. Efficacy and safety of abatacept, a T-cell modulator, in a randomised, doubleblind, placebo-controlled, phase III study in psoriatic arthritis. Annals of the Rheumatic Diseases 76, 1550-1558, doi:10.1136/annrheumdis-2016-210724 (2017).

59 Song, I. H. et al. Treatment of active ankylosing spondylitis with abatacept: an open-label, 24-week pilot study. Ann Rheum Dis 70, 1108-1110, doi:10.1136/ard.2010.145946 (2011).

60 Sandborn, W. J. et al. Abatacept for Crohn's Disease and Ulcerative Colitis. Gastroenterology 143, 62-69.e64, doi:10.1053/j.gastro.2012.04.010 (2012).

61 European Medicines Agency. Entyvio (Vedolizumab) Annex I Summary of product characteristics, $<$ https://www.ema.europa.eu/en/documents/product-information/entyvio-epar-productinformation en.pdf> (2019).

62 U.S. Food \& Drug Administration. Entyvio (Vedolizumab) Supplementary label SUPPL-24, <https://www.accessdata.fda.gov/drugsatfda docs/label/2019/125476s024lbl.pdf> (2019).

63 Feagan, B. G. et al. Efficacy of Vedolizumab in Fistulising Crohn's Disease: Exploratory Analyses of Data from GEMINI 2. Journal of Crohn's and Colitis 12, 621-626, doi:10.1093/ecco-jcc/jjy019 (2018). Varkas, G. et al. An induction or flare of arthritis and/or sacroiliitis by vedolizumab in inflammatory bowel disease: a case series. Ann Rheum Dis 76, 878-881, doi:10.1136/annrheumdis-2016-210233 (2017).

65 Wendling, D. et al. Arthritis occurrence or reactivation under Vedolizumab treatment for inflammatory bowel disease. A four cases report. Joint Bone Spine 85, 255-256, doi:10.1016/j.jbspin.2017.01.012 (2018).

66 Tamanini, S. et al. Letter to editor: New onset/recurrence of inflammatory arthralgia/spondyloarthritis in patients treated with vedolizumab for intestinal bowel disease. Clin Rheumatol 38, 609-610, doi:10.1007/s10067-018-4357-y (2019).

67 Orlando, A. et al. Clinical benefit of vedolizumab on articular manifestations in patients with active spondyloarthritis associated with inflammatory bowel disease. annrheumdis-2016, doi:10.1136/annrheumdis-2016-211011 (2017).

68 European Medicines Agency. Otezla (Apremilast) Annex I Summary of product characteristics, $<$ https://www.ema.europa.eu/en/documents/product-information/otezla-epar-productinformation en.pdf> (2019).

69 U.S. Food \& Drug Administration. Otezla (Apremilast) Supplementary label SUPPL-7, <https://www.accessdata.fda.gov/drugsatfda docs/label/2019/2054370rig1s007lbl.pdf> (2019). 
70 Pathan, E. et al. Efficacy and safety of apremilast, an oral phosphodiesterase 4 inhibitor, in ankylosing spondylitis. Ann Rheum Dis 72, 1475-1480, doi:10.1136/annrheumdis-2012-201915 (2013).

71 European Medicines Agency. Xeljanz (Tofacitinib) Annex I Summary of product characteristics, $<$ https://www.ema.europa.eu/en/documents/product-information/xeljanz-epar-productinformation en.pdf> (2019).

72 U.S. Food \& Drug Administration. Xeljanz (Tofacitinib) Supplementary label SUPPL-25, <https://www.accessdata.fda.gov/drugsatfda docs/label/2019/203214s025lbl.pdf> (2019).

73 Van Der Heijde, D. et al. Tofacitinib in patients with ankylosing spondylitis: a phase II, 16-week, randomised, placebo-controlled, dose-ranging study. Annals of the Rheumatic Diseases 76, 13401347, doi:10.1136/annrheumdis-2016-210322 (2017).

Papp, K. A. et al. Tofacitinib, an oral Janus kinase inhibitor, for the treatment of chronic plaque psoriasis: results from two randomized, placebo-controlled, phase III trials. 173, 949-961, doi:10.1111/bjd.14018 (2015).

Bachelez, H. et al. Tofacitinib versus etanercept or placebo in moderate-to-severe chronic plaque psoriasis: a phase 3 randomised non-inferiority trial. 386, 552-561, doi:10.1016/s01406736(14)62113-9 (2015).

Sandborn, W. J. et al. A phase 2 study of tofacitinib, an oral Janus kinase inhibitor, in patients with Crohn's disease. Clin Gastroenterol Hepatol 12, 1485-1493.e1482, doi:10.1016/j.cgh.2014.01.029 (2014).

77 Jansbøl, K. (KORA Det Nationale Institut for Kommuners og Regioners Analyse og Forskning, 2014).

78 Love, T. J. \& Kavanaugh, A. Golimumab in the treatment of psoriatic arthritis. Expert Review of Clinical Immunology 14, 893-898, doi:10.1080/1744666X.2018.1524755 (2018).

79 Kalliolias, G. D. \& Ivashkiv, L. B. TNF biology, pathogenic mechanisms and emerging therapeutic strategies. Nature Reviews Rheumatology 12, 49-62, doi:10.1038/nrrheum.2015.169 (2016). Mease, P. J. Biologic Therapy for Psoriatic Arthritis. Rheum Dis Clin North Am 41, 723-738, doi:10.1016/j.rdc.2015.07.010 (2015).

81 Papp, K. A. et al. Comparison of Adalimumab and Etanercept for the Treatment of Moderate to Severe Psoriasis: An Indirect Comparison Using Individual Patient Data from Randomized Trials. Value in Health 21, 1-8, doi:10.1016/j.jval.2017.05.025 (2018). Van den Brande, J. M. et al. Infliximab but not etanercept induces apoptosis in lamina propria Tlymphocytes from patients with Crohn's disease. Gastroenterology 124, 1774-1785, doi:10.1016/s0016-5085(03)00382-2 (2003). ten Hove, T., van Montfrans, C., Peppelenbosch, M. P. \& van Deventer, S. J. Infliximab treatment induces apoptosis of lamina propria T lymphocytes in Crohn's disease. Gut 50, 206-211, doi:10.1136/gut.50.2.206 (2002).

Atreya, R. et al. Antibodies against tumor necrosis factor (TNF) induce T-cell apoptosis in patients with inflammatory bowel diseases via TNF receptor 2 and intestinal CD14(+) macrophages. Gastroenterology 141, 2026-2038, doi:10.1053/j.gastro.2011.08.032 (2011). Mitoma, H. et al. Infliximab induces potent anti-inflammatory responses by outside-to-inside signals through transmembrane TNF-alpha. Gastroenterology 128, 376-392, doi:10.1053/j.gastro.2004.11.060 (2005).

86 Hawkes, J. E., Chan, T. C. \& Krueger, J. G. Psoriasis pathogenesis and the development of novel targeted immune therapies. Journal of Allergy and Clinical Immunology 140, 645-653, doi:10.1016/j.jaci.2017.07.004 (2017).

87 Deodhar, A. et al. Efficacy and safety of guselkumab in patients with active psoriatic arthritis: a randomised, double-blind, placebo-controlled, phase 2 study. The Lancet 391, 2213-2224, doi:10.1016/s0140-6736(18)30952-8 (2018). 
Girolomoni, G. et al. The role of IL-23 and the IL-23/TH 17 immune axis in the pathogenesis and treatment of psoriasis. Journal of the European Academy of Dermatology and Venereology 31, 1616-1626, doi:10.1111/jdv.14433 (2017).

89 Griffiths, C. E. M. et al. Comparison of Ustekinumab and Etanercept for Moderate-to-Severe Psoriasis. New England Journal of Medicine 362, 118-128, doi:10.1056/nejmoa0810652 (2010). Reich, K. et al. Efficacy and safety of guselkumab, an anti-interleukin-23 monoclonal antibody, compared with adalimumab for the treatment of patients with moderate to severe psoriasis with randomized withdrawal and retreatment: Results from the phase III, double-blind, p. Journal of the American Academy of Dermatology 76, 418-431, doi:10.1016/j.jaad.2016.11.042 (2017).

91 Reich, K. et al. Risankizumab compared with adalimumab in patients with moderate-to-severe plaque psoriasis (IMMvent): a randomised, double-blind, active-comparator-controlled phase 3 trial. The Lancet 394, 576-586, doi:10.1016/s0140-6736(19)30952-3 (2019).

92 Reich, K. et al. Tildrakizumab versus placebo or etanercept for chronic plaque psoriasis (reSURFACE 1 and reSURFACE 2): results from two randomised controlled, phase 3 trials. Lancet 390, 276-288, doi:10.1016/s0140-6736(17)31279-5 (2017).

93 Gossec, L. et al. EULAR recommendations for the management of psoriatic arthritis with pharmacological therapies: 2019 update. Ann Rheum Dis 79, 700-712, doi:10.1136/annrheumdis2020-217159 (2020).

94 Taurog, J. D., Chhabra, A. \& Colbert, R. A. Ankylosing Spondylitis and Axial Spondyloarthritis. New England Journal of Medicine 374, 2563-2574, doi:10.1056/nejmra1406182 (2016).

95 Nicola, S., Rolla, G., Monti, R. \& Brussino, L. Treatment of psoriatic arthritis with secukinumab: a case series. J Dermatolog Treat 29, 6-8, doi:10.1080/09546634.2018.1527994 (2018).

96 Chaplin, S. Ixekizumab: targeting plaque psoriasis and psoriatic arthritis. Prescriber 30, 37-38, doi:10.1002/psb.1743 (2019).

97 Mease, P. J. et al. Improvement in Psoriasis Signs and Symptoms Assessed by the Psoriasis Symptom Inventory with Brodalumab Treatment in Patients with Psoriatic Arthritis. J Rheumatol 43, 343-349, doi:10.3899/jrheum.150182 (2016).

98 Lebwohl, M. et al. Phase 3 Studies Comparing Brodalumab with Ustekinumab in Psoriasis. N Engl J Med 373, 1318-1328, doi:10.1056/NEJMoa1503824 (2015).

99 Griffiths, C. E. et al. Comparison of ixekizumab with etanercept or placebo in moderate-to-severe psoriasis (UNCOVER-2 and UNCOVER-3): results from two phase 3 randomised trials. Lancet 386, 541-551, doi:10.1016/s0140-6736(15)60125-8 (2015).

100 Langley, R. G. et al. Secukinumab in Plaque Psoriasis - Results of Two Phase 3 Trials. New England Journal of Medicine 371, 326-338, doi:10.1056/nejmoa1314258 (2014).

101 Reich, K. et al. Comparison of ixekizumab with ustekinumab in moderate-to-severe psoriasis: 24week results from IXORA-S, a phase III study. Br J Dermatol 177, 1014-1023, doi:10.1111/bjd.15666 (2017).

102 Reich, K. et al. Guselkumab versus secukinumab for the treatment of moderate-to-severe psoriasis (ECLIPSE): results from a phase 3, randomised controlled trial. The Lancet 394, 831-839, doi:https://doi.org/10.1016/S0140-6736(19)31773-8 (2019).

103 Abbvie. (2020).

104 Blauvelt, A. et al. A head-to-head comparison of ixekizumab vs. guselkumab in patients with moderate-to-severe plaque psoriasis: 12-week efficacy, safety and speed of response from a randomized, double-blinded trial. Br J Dermatol, doi:10.1111/bjd.18851 (2019).

105 Nash, P. et al. Ixekizumab for the treatment of patients with active psoriatic arthritis and an inadequate response to tumour necrosis factor inhibitors: results from the 24-week randomised, double-blind, placebo-controlled period of the SPIRIT-P2 phase 3 trial. Lancet 389, 2317-2327, doi:10.1016/s0140-6736(17)31429-0 (2017). 
Running title: SPEED-UP treatment

106 Mclnnes, I. B. et al. Secukinumab versus adalimumab for treatment of active psoriatic arthritis (EXCEED): a double-blind, parallel-group, randomised, active-controlled, phase $3 \mathrm{~b}$ trial. The Lancet 395, 1496-1505, doi:10.1016/S0140-6736(20)30564-X (2020).

107 Gordon, K. B. et al. Phase 3 Trials of Ixekizumab in Moderate-to-Severe Plaque Psoriasis. New England Journal of Medicine 375, 345-356, doi:10.1056/nejmoa1512711 (2016).

108 Lubrano, E., Scriffignano, S. \& Perrotta, F. M. Abatacept for the treatment of psoriatic arthritis. Expert Rev Clin Immunol 14, 899-905, doi:10.1080/1744666x.2018.1527218 (2018).

109 Kennedy, L. B. \& Salama, A. K. S. A Review of Immune-Mediated Adverse Events in Melanoma. Oncology and Therapy 7, 101-120, doi:10.1007/s40487-019-0096-8 (2019).

110 Deeks, E. D. Apremilast: A Review in Psoriasis and Psoriatic Arthritis. Drugs 75, 1393-1403, doi:10.1007/s40265-015-0439-1 (2015).

111 Kragstrup, T. W. et al. IL-12/IL-23p40 identified as a downstream target of apremilast in ex vivo models of arthritis. Therapeutic Advances in Musculoskeletal Disease 11, 1759720X1982866, doi:10.1177/1759720x19828669 (2019).

112 Dodington, D. W., Desai, H. R. \& Woo, M. JAK/STAT - Emerging Players in Metabolism. Trends in Endocrinology \& Metabolism 29, 55-65, doi:10.1016/j.tem.2017.11.001 (2018).

113 Kragstrup, T. W. et al. The IL-20 Cytokine Family in Rheumatoid Arthritis and Spondyloarthritis. Frontiers in immunology 9, 2226-2226, doi:10.3389/fimmu.2018.02226 (2018).

114 Papp, K. et al. Phase 2 Trial of Selective Tyrosine Kinase 2 Inhibition in Psoriasis. New England Journal of Medicine 379, 1313-1321, doi:10.1056/nejmoa1806382 (2018). 
Running title: SPEED-UP treatment

11 Tables

11.1.1 Table 1. Disease entities in the SPEED-UP spectrum and treatment options.

\begin{tabular}{|c|c|c|c|c|c|c|c|c|c|c|c|c|c|c|c|c|c|}
\hline & Target & & 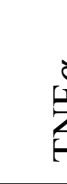 & & & $\frac{\infty}{\delta}$ & $\stackrel{?}{\mathfrak{t}}$ & & 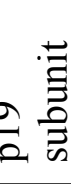 & & $\overleftrightarrow{r}$ & & 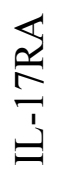 & $\begin{array}{l}0 \\
\infty \\
\infty \\
0 \\
0\end{array}$ & $\frac{\hat{o}}{\delta}$ & $\stackrel{+}{\overrightarrow{0}}$ & $\begin{array}{l}\stackrel{m}{\Xi} \\
\frac{\Delta}{\Xi}\end{array}$ \\
\hline & Drug & 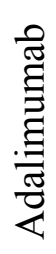 & 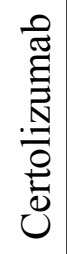 & 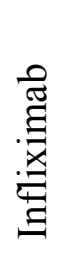 & 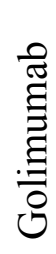 & 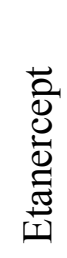 & 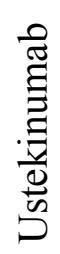 & 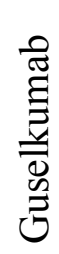 & 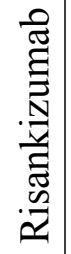 & 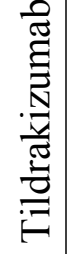 & 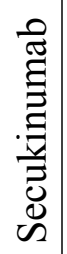 & 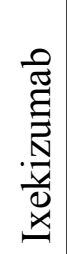 & 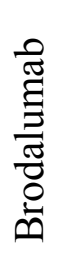 & 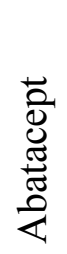 & 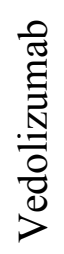 & 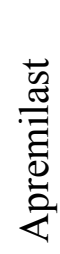 & 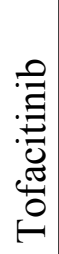 \\
\hline $\mathrm{Cn}$ & $\mathrm{R}-\mathrm{axSpA}$ & $* \neq$ & $* \neq$ & $* \neq$ & $* \neq$ & $* \neq$ & & & & & $* \neq$ & $* \neq$ & & & & & \\
\hline аXSPA & $\mathrm{Nr}-\mathrm{axSpA}$ & $*$ & $* \neq$ & & $*$ & $*$ & & & & & $* \neq$ & $* \neq$ & & & & & \\
\hline & Plaque & $*_{\ddagger}$ & $* \neq$ & $* \neq$ & & $* \neq$ & $* \neq$ & $* \neq$ & $* \neq$ & $* \neq$ & $* \neq$ & $* \neq$ & $* \neq$ & & & $* \neq$ & \\
\hline F Sotiasis & Nail & $* \neq$ & & & & & * & * & * & & * & * & $*$ & & & $*$ & \\
\hline & $\begin{array}{l}\text { Peripheral } \\
\text { arthritis }\end{array}$ & $* \neq$ & $* \neq$ & $* \neq$ & $* \neq$ & $* \neq$ & $* \neq$ & $\neq$ & & & $* \neq$ & $* \neq$ & & $* \neq$ & & $* \neq$ & $* \neq$ \\
\hline & Axial arthritis & & & & & & & & & & & & & & & & \\
\hline PsA & Entesitis & & & & & & & & & & & & & & & & \\
\hline & Dactylitis & & & & & & & & & & & & & & & & \\
\hline & $\begin{array}{l}\text { Radiographic } \\
\text { progression }\end{array}$ & $* \neq$ & $*$ & $*_{\neq}$ & $*$ & $* \neq$ & $* \neq$ & & & & $*$ & $*$ & & $*$ & & & \\
\hline & Crohn's disease & $* \neq$ & $\neq$ & $* \neq$ & & & $* \neq$ & & & & & & & & $* \neq$ & & \\
\hline IBD & $\begin{array}{l}\text { Fistulating } \\
\text { Crohn's } \\
\text { disease }\end{array}$ & & & $*$ & & & & & & & & & & & & & \\
\hline & $\begin{array}{l}\text { Ulcerative } \\
\text { colitis }\end{array}$ & $* \neq$ & & $* \neq$ & $* \neq$ & & $* \neq$ & & & & & & & & $* \neq$ & & $* \neq$ \\
\hline Uveitis & & $* \neq$ & & & & & & & & & & & & & & & \\
\hline
\end{tabular}

\begin{tabular}{|c|c|}
\hline Color code & Description \\
\hline Green & EMA (*) or FDA ( $¥)$ approved \\
& $\begin{array}{r}\text { Secondary endpoints are likewise green if mentioned } \\
\text { anywhere in the approval document. }\end{array}$ \\
\hline Yellow & Primary endpoints met in $\geq$ Phase 2 studies. \\
& Phase 2 or 3 in which significant results concerning either \\
& primary or secondary endpoints. \\
\hline
\end{tabular}


Running title: SPEED-UP treatment

\begin{tabular}{|c|c|}
\hline Red & $\begin{array}{c}\text { Contraindicated or to be used with caution } \\
\text { Tagged in this category if directly mentioned as a } \\
\text { contraindication, or if anywhere else in the approval } \\
\text { document mentioned as an inducer of severe adverse effects }\end{array}$ \\
\hline Dark grey & $\begin{array}{r}\text { Primary endpoint not met in } \geq \text { Phase } 2 \text { studies } \\
\text { Tagged in this category if the results describe insignificant } \\
\text { results }(p>0,05) . \\
\text { No data or below phase } 2 \\
\text { All phase } 1 \text { studies were rejected. }\end{array}$ \\
\hline
\end{tabular}

Brüner $M$ et al 
12 Figures and legends

A

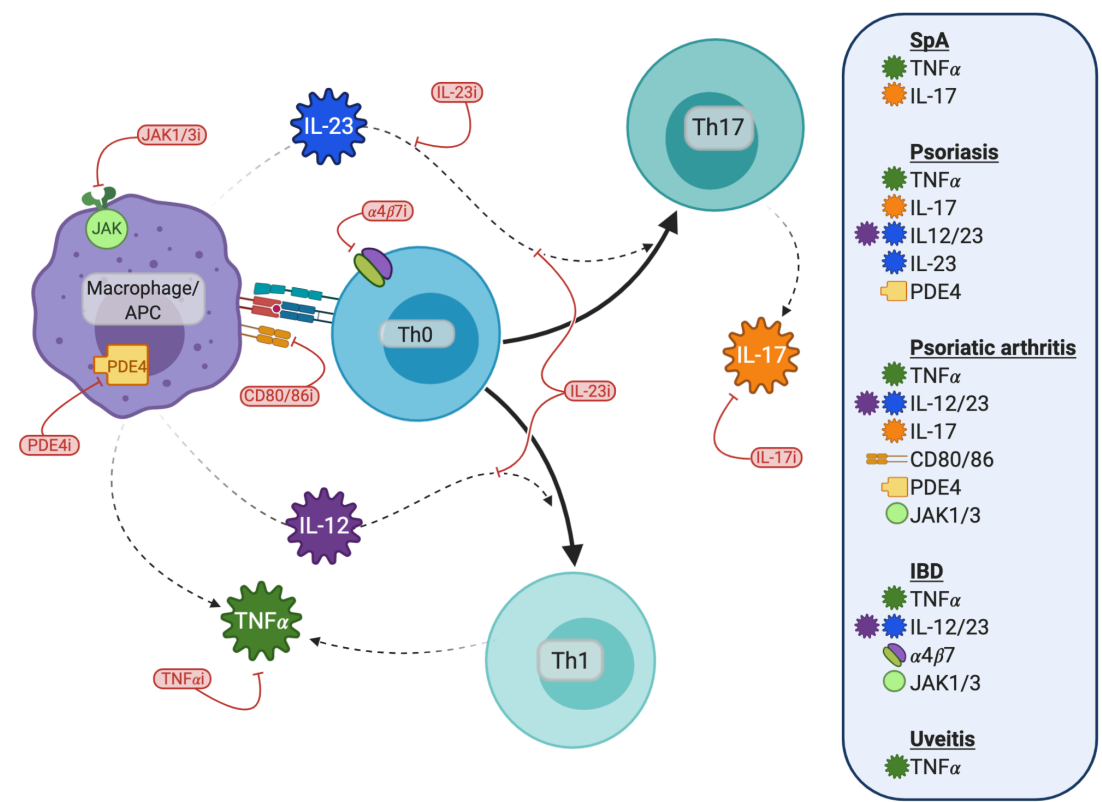

B

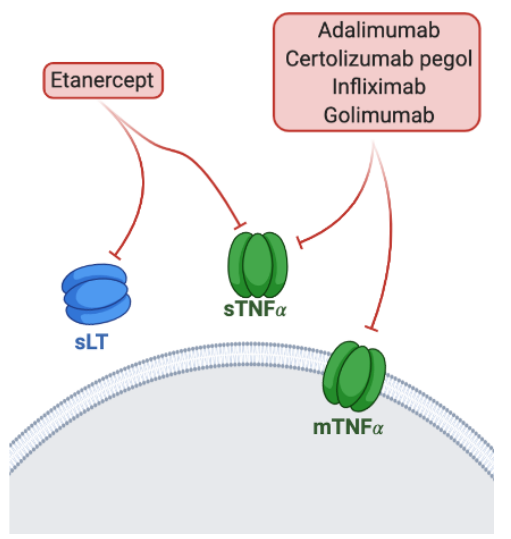

C
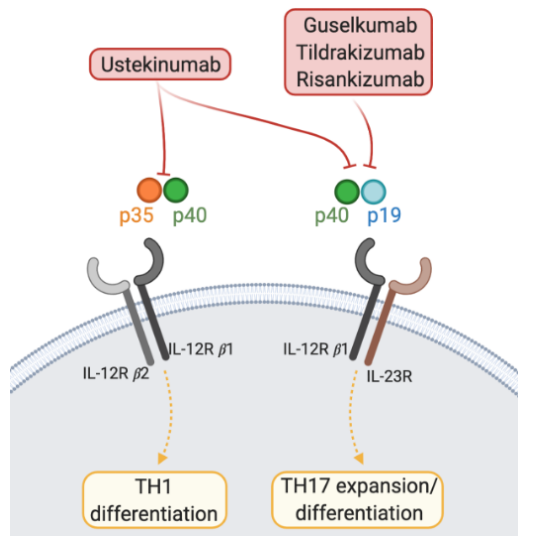

C

Secukinumab

D
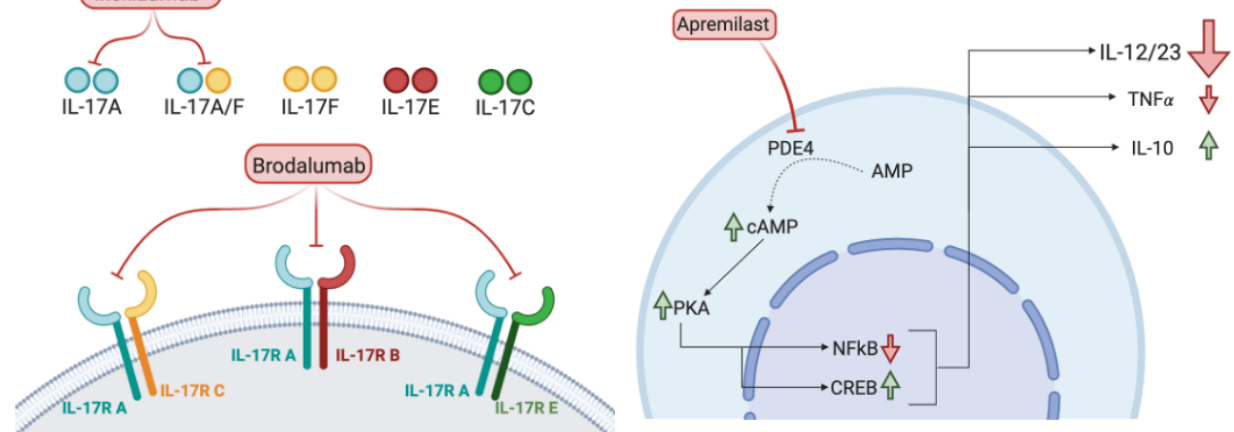

E
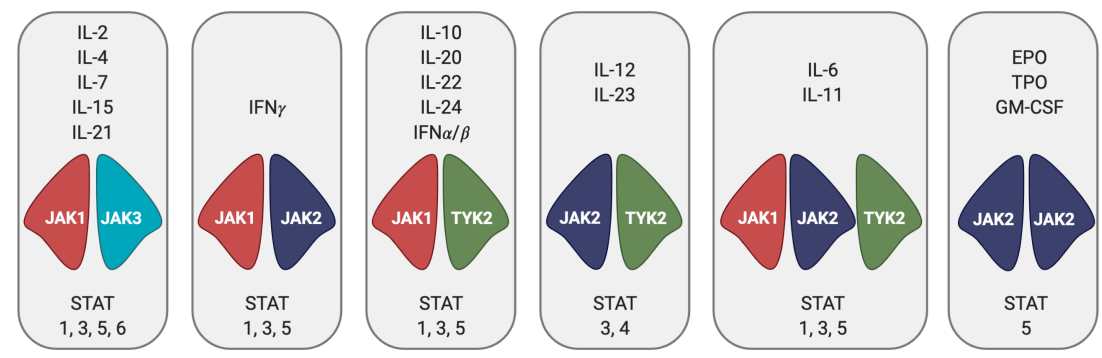
Running title: SPEED-UP treatment

Figure 1. A. Illustration of the different treatment targets of drugs with at least one indication by EMA or FDA in the SPEED-UP disease spectrum. B. Illustration of the different TNF inhibitors. C. Illustration of the different IL-23 inhibitors. D. Illustration of the different IL-17 inhibitors. E. Illustration of the mode of action of apremilast including downstream effects on cytokine expression. F. Cytokines signaling via JAK/STAT pathways.

12.1.1 